\title{
FACTORES ASOCIADOS A MALFORMACIONES CONGÉNITAS
}

\author{
Félix Dasio Ayala Peralta1,2,a, Enrique Guevara Ríos ${ }^{2, b}$, César Carranza Asmat 2,b , Antonio Luna \\ Figueroa ${ }^{2, b}$, Marcos Espinola-Sánchez ${ }^{2, c}$, Augusto Racchumí Vela ${ }^{2, d}$, Melisa Mejico Caja ${ }^{2, e}$, Sabrina \\ Morales Alvarado ${ }^{1, f}$, Vanessa Valdivieso Oliva ${ }^{1, g}$, Bertha Nathaly Reyes Serrano ${ }^{1,9}$, Adriana Josefina \\ Barbaggelata Huaraca $^{1, g}$, Katherin Faviola Moreno Reyes ${ }^{1, h}$.
}

\section{RESUMEN}

Objetivo: Identificar los factores de riesgo obstétricos y perinatales asociados a recien nacidos con malformaciones congénitas (MC) en gestantes atendidas en el Instituto Nacional Materno Perinatal durante el periodo 2018. Material y Métodos: Estudio observacional, retrospectivo, corte transversal en mujeres hospitalizadas de enero a diciembre 2018 que cumplieron con criterios de inclusión. Las variables de estudio fueron: edad materna, edad gestacional, tipo de parto, peso al nacer, puntuación Apgar al nacer, tipo de MC y supervivencia. Se utilizó análisis estadístico para cálculo de prevalencias y proporciones. Se aplicó la prueba de Mann-Whitney y Chi-cuadrado. Para estimar las curvas de sobrevida se usó el Método de Kaplan Meier. Resultados: Se enrolaron 340 casos que representan el 1,9\% de prevalencia de MC. La tasa de mortalidad neonatal específica de MC fue $8,1 \times 1000$ nv. Corresponden $7,9 \%$ a mujeres adolescentes y $29,7 \%$ edad materna avanzada. Culminaron por cesárea en $65,9 \%$. El 33,5\% corresponden a recién nacido (RN) con bajo peso al nacer $(p<0,05)$. El $68,5 \%$ fueron de edad gestacional a término y $31,5 \%$ pretérmino; de ellos, fallecieron $54,2 \%$ y $45,8 \%$ RN pretérmino y a término respectivamente $(p<0,05)$. Correspondió Apgar bajo al nacer al minuto menor de puntaje 7 al $37,9 \%$ y a los 5 minutos $20,8 \%(p<0,05)$. Según tipo de MC, en madres adolescentes predominan macrocefalia con $44,4 \%$, testículo no descendido $22,2 \%$, hidrocefalia $11,1 \%$, entre otras; en edad media, otros síndromes de malformaciones congénitas de causas exógenas con $15,4 \%$, seguida MC renal $11 \%$; anemia congénita y macrocefalia $7,7 \%$; entre otras; y en edad materna avanzada predomina el Síndrome de Down con 26,4\%, seguida de otros síndromes de malformaciones congénitas debidas a causas exógenas con $15,1 \%$; malformaciones múltiples con $7,1 \%$ entre otras.

La probabilidad de supervivencia de RN sexo femenino es mayor que el masculino alcanzando por encima del $60 \%$ después de 20 días; y los RN de madres de edad media y edad materna avanzada sobreviven mayor que de las madres adolescentes alcanzando por encima del $62 \%$ después de 15 días de nacido. Conclusión: Recien nacido pretérmino con bajo peso al nacer y Apgar bajo al nacer tuvieron asociación estadística significativa; asimismo, el Sindrome de Down fue la MC predominante en edad materna avanzada.

Palabras Claves: Factores de riesgo, malformación congénita, edad materna avanzada (Fuente: DeCS BIREME).

\section{FACTORS ASSOCIATED WITH CONGENITAL MALFORMATIONS}

\begin{abstract}
Objective: To identify the obstetric and perinatal risk factors associated to newborns with congenital malformations (CM) in pregnant women treated at the National Maternal Perinatal Institute during the 2018 period. Material and Methods: Observational, retrospective, cross-sectional study in hospitalized women in January as of December 2018 that met inclusion criteria. The study variables were: maternal age, gestational age, type of birth, birth weight, Apgar at birth score, type of MC and survival. Statistical analysis was used to calculate prevalences and proportions. The Mann-Whitney and Chi-square test was applied. The Kaplan Meier Method was used to estimate the survival curves. Results: 340 cases were enrolled representing $1,9 \%$ prevalence of MC. The specific neonatal mortality rate of MC was $8.1 \times 1000 \mathrm{nv}$. They correspond 7,9\% to adolescent women and $29,7 \%$ advanced maternal age. They culminated by caesarean section in $65,9 \% .33,5 \%$ correspond to newborn (RN) with low birth weight $(p<0,005) .68,5 \%$ were of term gestational age and $31,5 \%$ preterm; of them, $54,2 \%$ and $45,8 \%$ preterm and term RN died respectively $(p<0,005)$. Corresponded low Apgar at birth at the lowest minute of score 7 to $37,9 \%$ and at 5 minutes $20,8 \%(p<0,005)$. According to type of MC, macrocephaly with $44,4 \%$ predominates in adolescent mothers, $22,2 \%$ undescended testis, $11,1 \%$ hydrocephalus, among others; in middle age, other syndromes of
\end{abstract}

\section{Universidad Norbert Wiener. Lima-Perú}

Instituto Nacional Materno Perinatal. Lima Perú

Médico GínecoObstetra. Profesor Ordinario de Facultad de Medicina de Universidad Nacional Mayor de San Marcos y Ciencias de la Salud de Universidad Norbert Wiener. Lima-Perú. Código ORCID http://orcid.org/0000-0002-2830-3789

Médico GínecoObstetra. Profesor de Facultad de Medicina Universidad

Nacional Mayor de San Marcos. Lima-Perú.

Médico en Investigación Clínica. Lima-Perú

d Lic. en Estadística. Asistente de Unidad de investigación, Lima-Perú.

e Lic. en Administración. Asistente de Unidad de investigación. Lima-Perú.

Lic. en Obstetricia. Directora de la Escuela Académico Profesional de Obstetricia. Universidad Norbert Wiener. Lima-Perú.

$g$ Lic. en Obstetricia. Docente de la Escuela Académico Profesional de Obstetricia. Lima-Perú.

Internado en Obstetricia. Escuela Académico Profesional de Obstetricia. Lima-Perú.

Citar como: Ayala FD, Guevara E, Carranza C, Luna A, Espinola-Sanchez M, Racchumí A, Mejico M, Morales S, Valdivieso V, Reyes BN, Barbaggelata AJ, Moreno KF. Factores asociados a malformaciones congénitas. Rev Peru Investig Matern Perinat. 2019;8(4): 30-40 DOI https://doi.org/10.33421/inmp.2019171 
congenital malformations of exogenous causes with $15,4 \%$, followed by $11 \%$ renal MC; congenital anemia and macrocephaly $7,7 \%$; among other; and in advanced maternal age Down Syndrome predominates with $26,4 \%$, followed by other congenital malformation syndromes due to exogenous causes with $15,1 \%$; multiple malformations with $7,1 \%$ among others. The probability of female RN survival is greater than the male reaching over $60 \%$ after 20 days; and the RN of mothers of middle age and advanced maternal age survive longer than of teenage mothers reaching over $62 \%$ after 15 days of birth. Conclusion: Preterm newborn with low birth weight and low Apgar at birth had significant statistical association; also, Down Syndrome was the predominant MC in advanced maternal age.

Keywords: Risk factors; congenital malformation; advanced maternal age (Source: MeSH NLM).

\section{INTRODUCCIÓN}

En la actualidad, las malformaciones congénitas (MC) continúan siendo uno de los grandes problemas de la salud perinatal debido a la gran morbimortalidad fetal y neonatal que ésta lleva asociada.

La Organización Mundial de la (OMS) calcula que, cada año, 276.000 recién nacidos fallecen durante los primeros 28 días de vida por malformaciones congénitas; y constituyen la segunda causa de mortalidad infantil en América del Sur ${ }^{1,2}$. En el Instituto Nacional Materno Perinatal durante el periodo 2018 la MC ocupa el primer lugar como causa de mortalidad en etapa neonatal ${ }^{3}$.

Las MC, son también conocidas como anomalías congénitas, trastornos congénitos, defectos de nacimiento o enfermedades congénitas ${ }^{1}$. Se pueden definir como alteraciones morfológicas, estructurales, funcionales o moleculares, de los órganos, sistemas o partes del cuerpo que se producen durante la vida intrauterina, y son causados por factores genéticos, ambientales o ambos; pueden ser detectadas en la etapa prenatal, al momento del nacimiento o posteriores durante el transcurso de la vida $^{1,2}$.

En general las MC afectan entre $2-5 \%$ de nacimientos ${ }^{4} ; \mathrm{y}$ en la mayoría de las poblaciones, tienen una frecuencia aproximada a $3 \%$ en recién nacidos vivos y de $10 \%$ a $15 \%$ en recién nacidos muertos ${ }^{5,6}$. Se han estimado que el $10 \%$ de las malformaciones son atribuibles a factores ambientales, $25 \%$ a factores genéticos y $65 \%$ a factores desconocidos probablemente de orden multifactorial ${ }^{2,7}$.

Cabe destacar que las MC graves más frecuentes son las malformaciones cardíacas, los defectos del tubo neural y el síndrome de Down ${ }^{1,2}$.

El tipo más frecuente de MC estructural son las cardiopatías congénitas, que afectan a $1 \%$ de recién nacidos (RN) mientras que las anomalías metabólicas aparecen en 1 de cada $3.500 \mathrm{RN}^{2,5}$. Las malformaciones mayores más frecuentemente encontradas en Sudamérica son las alteraciones cardiacas (28 por $10.000 \mathrm{NV}$ ), los defectos de cierre de tubo neural (24 por $10.000 \mathrm{NV}$ ), síndrome de Down (16 por $10.000 \mathrm{NV}$ ), labio/paladar hendido (15 por $10.000 \mathrm{NV}$ ) y los defectos de pared abdominal (4 por $10.000 \mathrm{NV})^{2,5}$.

Según reportes actuales ${ }^{8}$, los factores de riesgo evidenciados para MC incluyen: edad materna avanzada, bajo peso y talla para la edad gestacional, restricción del crecimiento fetal, antecedentes de malformaciones congénitas en la familia, factores físicos, exposición materna a agroquímicos y enfermedades agudas de la madre en el primer trimestre del embarazo $0^{9,10}$.

La edad materna avanzada es considerada un factor de riesgo para la presentación de alteraciones cromosómicas numéricas (aneuploidías), en especial trisomías como el síndrome Down, síndrome Patau o síndrome Edwards ${ }^{11,12}$.

Por otro lado, reportes recientes en Estados Unidos ${ }^{11}$, indican la prevalencia estimada del síndrome de Down de aproximadamente $12 / 10,000$ nacidos vivos y $7 / 10,000$ en población general ${ }^{13}$; y en Europa 11/10,000 nacimientos ${ }^{11}$;

Tal como reporta Loane $\mathrm{M}$ et $\mathrm{al}^{11}$, sobre tendencias de veinte años en la prevalencia del síndrome de Down y otras trisomías en Europa, la prevalencia total por cada 10,000 nacimientos fue de 22.0 para la trisomía $21,5.0$ para la trisomía 18 y 2.0 para la trisomía 13; la prevalencia de nacimientos vivos fue de 11.2 para la trisomía 21, 1.04 para la trisomía 18 y 0.48 para la trisomía 13.

Por otro lado, los factores de riesgo para espina bífida ${ }^{14}$ incluyen: los antecedentes familiares de espina bífida 0 anencefalia pueden ser el factor de riesgo más fuerte, ingesta materna inadecuada de ácido fólico o ácido fólico natural durante la periconcepción y el embarazo temprano, diabetes materna pregestacional y exposición prenatal a anticonvulsivos como el ácido valproico o la carbamazepina.

Según reporte de Khoshnood B et al (15) en Europa la prevalencia de defecto de tubo neural fue 9,11 por 10 000 nacimientos y en Brasil fue 4,73 por 1000 partos $^{16}$.

Es importante recomendar que las medidas preventivas para $\mathrm{MC}$ es la suplementación con folato periconcepcional durante 3 meses previos a la concepción y el primer trimestre del embarazo para la prevención de defectos del tubo neural. Dicha suplementación periconcepcional materna con ácido fólico solo o con multivitaminas que contienen ácido fólico reducen el riesgo de espina bífida y anencefalia hasta en un $70 \%{ }^{14}$.

El objetivo de la investigación fue identificar los factores obstétricos antenatales asociados a recién nacidos con malformaciones congénitas atendidas durante el periodo de enero a diciembre del 2018 en el Instituto Nacional Materno Perinatal de Lima-Perú.

\section{MATERIALES Y MÉTODOS}

Población de estudio: Se desarrolló un diseño observacional, descriptiva, de corte transversal en todas mujeres que tuvieron recién nacido vivo con 
malformaciones congénitas diagnosticadas al momento del nacimiento, y que nacieron por parto vía vaginal o cesárea, con edad gestacional a término o pretérmino, atendidas en el Instituto Nacional Materno Perinatal durante el periodo de enero a diciembre del 2018. Los criterios de inclusión fueron recién nacidos con malformaciones congénitas. Se enrolaron 340 casos a los cuales se estudió las siguientes variables: edad materna, paridad, mujeres con historia de malformación congénita previa, número de controles prenatales, tipo de ingesta de medicamentos, edad gestacional al momento de ingreso y del parto, tipo de parto, peso al nacer, puntuación Apgar al nacer y tipo de malformación congénita. Este estudio fue aprobado por el Comité de Ética institucional.

Recolección de datos: Todas las mujeres fueron identificadas con las variables descritas según la historia obstétrica. Los datos fueron llevados a una ficha de recolección del estudio y registrados en un formulario de Google Forms ${ }^{\circledR}$ para homogenizar los datos y construir la base de datos del estudio.

Definiciones operacionales: La malformación congénita es definida como alteraciones morfológicas, estructurales, funcionales o moleculares, que se identifican al momento del nacimiento.

Se han incluido todos los recién nacidos vivos, con diagnóstico de $\mathrm{MC}$ al nacer o hasta el egreso hospitalario con diagnóstico confirmado por clínica o laboratorio de un defecto congénito (funcionales metabólicos, funcionales sensoriales o malformaciones congénitas).

Además, se utilizó la lista de chequeo STROBE (STrengthening the Reporting of OBservational studies in Epidemiology) para la formulación de estudios observacionales.

Análisis estadístico: El análisis estadístico se realizó en dos fases. La primera comprendió la estadística descriptiva en la que se analizó la frecuencia de los datos categóricos; se analizó las variables cualitativas según prevalencias y comparaciones de proporciones (prueba de homogeneidad) mediante el estadístico de Chi cuadrado. En las variables numéricas se realizó el resumen mediante estadístico de posición y dispersión, además se aplicó la prueba de Mann-Whitney para las comparaciones en los grupos y para cuantificar la medida de fuerza de asociación se utilizó el cálculo de OR con un nivel de confianza del 95\%. La segunda fase comprendió la estadística analítica en la que se incluyó para estimar las Curvas de Sobrevida el Método de Kaplan Meier. Todo el análisis estadístico se realizó en el paquete estadístico IBM-SPSS versión 22,0 . El nivel de significancia estadística para la pruebas estadísticas fue un $p<0,05$.

Consideraciones éticas: La investigación fue aprobada por comité de ética institucional y de la Universidad Norbert Wiener. No requirió consentimiento informado.

\section{RESULTADOS}

Durante el periodo 2018 , en el Instituto Nacional Materno Perinatal de Lima, ocurrieron en total 18175 nacidos vivos, de los cuales, según grupo etáreo correspondió a gestantes adolescentes $<20$ años $11.4 \%$, edad media de 20 a 34 años $67.6 \%$ y edad materna avanzada $\geq$ 35 años $21.0 \%^{3,17}$. Se enrolaron 340 casos de egresos hospitalarios de recien nacidos vivos con diagnosticos de malformaciones congénitas que representan el $1,9 \%$ de prevalencia de MC.

Asimismo, en el servicio de neonatología, durante el mismo periodo fallecieron 287 neonatos, de los cuales 141 casos $(49,1 \%)$ corresponden como causales a malformaciones congenitas ${ }^{17}$; cuya tasa de mortalidad neonatal específica de MC arroja 8,1 por 1000 rnv. Siendo las malformaciones congénitas prevalentes en orden de prelación correspondiente al sistema digestivo, nervioso, cardiovascular, renal, cromosomopatía, musculoesquelético y respiratorio ${ }^{3,17}$.

Tabla 1. Características obstétricas y perinatales como factores de riesgo para malformación congénita y resultado perinatalInstituto Nacional Materno Perinatal - 2018

\begin{tabular}{|c|c|c|c|c|c|c|c|c|c|}
\hline \multirow{3}{*}{\multicolumn{2}{|c|}{$\begin{array}{c}\text { Caracteristicas maternas y } \\
\text { perinatales }\end{array}$}} & \multirow{2}{*}{\multicolumn{2}{|c|}{ Total }} & \multicolumn{4}{|c|}{ Resultado perinatal } & \multirow{3}{*}{ p } & \multirow{3}{*}{ OR e IC al $95 \%$} \\
\hline & & & & \multicolumn{2}{|c|}{ Muerto } & \multicolumn{2}{|c|}{ Vivo } & & \\
\hline & & $\begin{array}{c}n \\
(340)\end{array}$ & $\%$ & n (118) & $\%$ & $\begin{array}{c}n \\
(222)\end{array}$ & $\%$ & & \\
\hline \multirow{3}{*}{$\begin{array}{l}\text { Edad de la } \\
\text { madre }\end{array}$} & $\begin{array}{l}\text { Mujer } \\
\text { Adolescentes }\end{array}$ & 27 & $7.9 \%$ & 13 & $11.0 \%$ & 14 & $6.3 \%$ & $>0.05$ & Ref \\
\hline & Edad media & 212 & $62.4 \%$ & 75 & $63.6 \%$ & 137 & $61.7 \%$ & $>0.05$ & $0.59(0.26-1.32)$ \\
\hline & $\begin{array}{l}\text { Edad materna } \\
\text { avanzada }\end{array}$ & 101 & $29.7 \%$ & 30 & $25.4 \%$ & 71 & $32.0 \%$ & $>0.05$ & $0.46(0.19-1.08)$ \\
\hline \multirow{2}{*}{ Vía de parto } & Cesarea & 224 & $65.9 \%$ & 81 & $68.6 \%$ & 143 & $64.4 \%$ & $>0.05$ & \multirow{2}{*}{-} \\
\hline & Parto Vaginal & 116 & $34.1 \%$ & 37 & $31.4 \%$ & 79 & $35.6 \%$ & $>0.05$ & \\
\hline \multirow{2}{*}{$\begin{array}{l}\text { Sexo del } \\
\text { recien nacido }\end{array}$} & Masculino & 193 & $56.8 \%$ & 66 & $55.9 \%$ & 127 & $57.2 \%$ & $>0.05$ & \multirow{2}{*}{ - } \\
\hline & Femenino & 147 & $43.2 \%$ & 52 & $44.1 \%$ & 95 & $42.8 \%$ & $>0.05$ & \\
\hline \multirow{3}{*}{$\begin{array}{l}\text { Peso del } \\
\text { recien nacido }\end{array}$} & Bajo peso & 114 & $33.5 \%$ & 70 & $59.3 \%$ & 44 & $19.8 \%$ & $<0.05$ & $5.69(3.46-9.35)$ \\
\hline & Peso normal & 215 & $63.2 \%$ & 47 & $39.8 \%$ & 168 & $75.7 \%$ & $<0.05$ & Ref \\
\hline & Macrosómico & 11 & $3.2 \%$ & 1 & $.8 \%$ & 10 & $4.5 \%$ & $>0.05$ & $0.36(0.04-2.86)$ \\
\hline
\end{tabular}




\begin{tabular}{|c|c|c|c|c|c|c|c|c|c|}
\hline \multirow{3}{*}{\multicolumn{2}{|c|}{$\begin{array}{c}\text { Caracteristicas maternas y } \\
\text { perinatales }\end{array}$}} & \multirow{2}{*}{\multicolumn{2}{|c|}{ Total }} & \multicolumn{4}{|c|}{ Resultado perinatal } & \multirow{3}{*}{ p } & \multirow{3}{*}{ OR e IC al $95 \%$} \\
\hline & & & & \multicolumn{2}{|c|}{ Muerto } & \multicolumn{2}{|c|}{ Vivo } & & \\
\hline & & $\begin{array}{c}n \\
(340)\end{array}$ & $\%$ & n (118) & $\%$ & $\begin{array}{c}n \\
(222)\end{array}$ & $\%$ & & \\
\hline \multirow{3}{*}{$\begin{array}{l}\text { Apgar al nacer } \\
\text { al minuto }\end{array}$} & $1-3$ & 67 & $19.7 \%$ & 60 & $50.8 \%$ & 7 & $3.2 \%$ & $<0.05$ & - \\
\hline & $4-6$ & 62 & $18.2 \%$ & 27 & $22.9 \%$ & 35 & $15.8 \%$ & $>0.05$ & $4.48(2.38-8.41)$ \\
\hline & $7-10$ & 211 & $62.1 \%$ & 31 & $26.3 \%$ & 180 & $81.1 \%$ & $<0.05$ & Ref \\
\hline \multirow{3}{*}{$\begin{array}{l}\text { Apgar al nacer } \\
\text { a los } 5 \mathrm{~min}\end{array}$} & $1-3$ & 45 & $13.2 \%$ & 44 & $37.3 \%$ & 1 & $.5 \%$ & $<0.05$ & - \\
\hline & $4-6$ & 26 & $7.6 \%$ & 25 & $21.2 \%$ & 1 & $.5 \%$ & $<0.05$ & - \\
\hline & $7-10$ & 269 & $79.1 \%$ & 49 & $41.5 \%$ & 220 & $99.1 \%$ & $<0.05$ & Ref \\
\hline \multirow{5}{*}{$\begin{array}{l}\text { Edad del } \\
\text { neonato }\end{array}$} & Media & & & \multicolumn{2}{|c|}{15.69} & \multicolumn{2}{|c|}{21.26} & & \\
\hline & Mediana & & & \multicolumn{2}{|c|}{11.00} & \multicolumn{2}{|c|}{15.00} & & \\
\hline & DS & & & \multicolumn{2}{|c|}{16.923} & \multicolumn{2}{|c|}{16.442} & $<0.05$ & \\
\hline & Mínimo & & & \multicolumn{2}{|c|}{1} & \multicolumn{2}{|c|}{4} & & \\
\hline & Máximo & & & \multicolumn{2}{|c|}{126} & \multicolumn{2}{|c|}{89} & & \\
\hline \multirow{5}{*}{$\begin{array}{l}\text { Perímero } \\
\text { cefalico }\end{array}$} & Media & & & \multicolumn{2}{|c|}{31.75} & \multicolumn{2}{|c|}{34.34} & & \\
\hline & Mediana & & & \multicolumn{2}{|c|}{32.25} & \multicolumn{2}{|c|}{34.20} & & \\
\hline & DS & & & \multicolumn{2}{|c|}{4.983} & \multicolumn{2}{|c|}{3.032} & $<0.05$ & \\
\hline & Mínimo & & & \multicolumn{2}{|c|}{20} & \multicolumn{2}{|c|}{25} & & \\
\hline & Máximo & & & \multicolumn{2}{|c|}{43} & & 8 & & \\
\hline & Media & & & & & & 62 & & \\
\hline & Mediana & & & & & & .00 & & \\
\hline $\begin{array}{l}\text { Talla del } \\
\text { recien nacido }\end{array}$ & DS & & & & & & 33 & $<0.05$ & \\
\hline & Mínimo & & & & & & 3 & & \\
\hline & Máximo & & & & & & 4 & & \\
\hline & Media & & & & & & 61 & & \\
\hline & Mediana & & & & & & 00 & & \\
\hline $\begin{array}{l}\text { Estadía total } \\
\text { en hospital }\end{array}$ & DS & & & & & & 142 & $<0.05$ & \\
\hline & Mínimo & & & & & & 1 & & \\
\hline & Máximo & & & & & & 5 & & \\
\hline
\end{tabular}

En la tabla 1 se evidencia que de los 340 casos estudiados prevalecen en la mayoría en gestantes de edad media y en los extremos de la vida corresponden 7,9\% (27 casos) a gestantes adolescentes y $29,7 \%$ (101 casos) a edad materna avanzada; de ellos, fallecieron el $11 \%$ de MC de mujeres adolescentes y $25,4 \%$ en edad materna avanzada. La vía de parto fue cesárea en $65,9 \%$ de mujeres con MC. El $56,8 \%$ de MC corresponden a sexo masculino. El 33,5\% corresponden a RN con MC de bajo peso al nacer, siendo este un factor de riesgo estadísticamente significativo para la muerte de un $\mathrm{RN}$ con $\mathrm{MC}(\mathrm{OR}=5.69)$ y $3,2 \%$ macrosomía fetal. El $68,5 \%$ (233 casos) fueron de edad gestacional a término y el $31,5 \%$ (107 casos) corresponden a pretérmino (factor de riesgo significativo con un OR=4.93); de ellos, fallecieron $54,2 \%$ y $45,8 \%$ pretérmino y a término respectivamente $(p<0,05)$ que es estadísticamente significativo. Correspondió Apgar al minuto menor de puntaje 7 al $37.9 \%$ y a los 5 minutos $20,8 \%(p<0,05)$ que son estadísticamente significativo el Apgar bajo al nacer. El resultado perinatal en relación a la edad del neonato tuvieron edad media de los neonatos vivos de 21.26 días (DS $\pm 16,44$ ) y de neonatos que fallecieron a la edad media de 15,69 (DS $\pm 16,92)$ que fue estadísticamente significativa $(p<0,05)$. La estancia hospitalaria fue de una media 13,6 (DS $\pm 15,14$ ) días para malformaciones congénitas que egresaron vivos y de 7,62 días (DS $\pm 11,97)$ de los MC fallecidos $(p<0,05)$ que fue estadísticamente significativa. 
Tabla 2. Clasificación para malformaciones congénitas según CIE 10. Instituto Nacional Materno Perinatal - 2018

\begin{tabular}{|c|c|c|}
\hline Clasificación según CIE 10 para malformaciones congénitas & $\mathbf{N}$ & Porcentaje \\
\hline Otros síndromes de malformaciones congénitas debidos a causas exógenas conocidas & 22 & $14.30 \%$ \\
\hline Síndrome de Down, no especificado & 17 & $11.00 \%$ \\
\hline Macrocefalia & 14 & $9.10 \%$ \\
\hline Malformación congénita del riñón, no especificada & 12 & $7.80 \%$ \\
\hline Otras anemias congénitas, no clasificadas en otra parte & 9 & $5.80 \%$ \\
\hline Hidrocéfalo congénito, no especificado & 6 & $3.90 \%$ \\
\hline Malformaciones congénitas múltiples, no clasificadas en otra parte & 6 & $3.90 \%$ \\
\hline Ausencia, atresia y estenosis congénita del recto, sin fístula & 5 & $3.20 \%$ \\
\hline Testículo no descendido, sin otra especificación & 5 & $3.20 \%$ \\
\hline Malformación congénita del sistema nervioso, no especificada & 4 & $2.60 \%$ \\
\hline Malformación congénita del corazón, no especificada & 4 & $2.60 \%$ \\
\hline Fisura del paladar con labio leporino & 3 & $1.90 \%$ \\
\hline Otras malformaciones congénitas del hígado & 3 & $1.90 \%$ \\
\hline Riñón poliquístico, autosómico recesivo & 3 & $1.90 \%$ \\
\hline Hidronefrosis congénita & 3 & $1.90 \%$ \\
\hline Otras malformaciones congénitas especificadas del (de los) miembro(s) & 3 & $1.90 \%$ \\
\hline Hidrocele congénito & 2 & $1.30 \%$ \\
\hline Otras deformidades congénitas de los pies & 2 & $1.30 \%$ \\
\hline Feto y recién nacido afectados por anormalidad no especificada de las membranas & 2 & $1.30 \%$ \\
\hline Malformaciones congénitas del cuerpo calloso & 2 & $1.30 \%$ \\
\hline Espina bífida & 2 & $1.30 \%$ \\
\hline Fisura del paladar duro y del paladar blando con labio leporino bilateral & 2 & $1.30 \%$ \\
\hline Ausencia, atresia y estenosis congénita del duodeno & 2 & $1.30 \%$ \\
\hline Ausencia, atresia y estenosis congénita del ano, sin fístula & 2 & $1.30 \%$ \\
\hline Hipospadias, no especificada & 2 & $1.30 \%$ \\
\hline Conducto arterioso permeable & 1 & $0.60 \%$ \\
\hline Síndrome del seno enfermo & 1 & $0.60 \%$ \\
\hline Hipotonía congénita & 1 & $0.60 \%$ \\
\hline Microcefalia & 1 & $0.60 \%$ \\
\hline Atresia de los agujeros de Magendie y de Luschka & 1 & $0.60 \%$ \\
\hline Otras anomalías hipoplásicas del encéfalo & 1 & $0.60 \%$ \\
\hline Espina bífida lumbar sin hidrocéfalo & 1 & $0.60 \%$ \\
\hline Fisura del paladar, sin otra especificación & 1 & $0.60 \%$ \\
\hline Labio leporino & 1 & $0.60 \%$ \\
\hline Testículo no descendido, unilateral & 1 & $0.60 \%$ \\
\hline Hiperplasia renal y riñón gigante & 1 & $0.60 \%$ \\
\hline Otras deformidades congénitas de la cadera & 1 & $0.60 \%$ \\
\hline Pie cavus & 1 & $0.60 \%$ \\
\hline Deformidad congénita de la columna vertebral & 1 & $0.60 \%$ \\
\hline Artrogriposis múltiple congénita & 1 & $0.60 \%$ \\
\hline Malformación congénita de miembro(s), no especificada & 1 & $0.60 \%$ \\
\hline Acondroplasia & 1 & $0.60 \%$ \\
\hline Total & 154 & $100.00 \%$ \\
\hline
\end{tabular}

En general, en la tabla 2 se aprecia la prevalencia de las distintas MC según CIE 10, que corresponden el $14,3 \%$ a otros síndromes de malformaciones congénitas de causas exógenas, seguida de síndrome de Down con $11 \%$, macrocefalia $9,4 \%$, malformación renal $7,8 \%$ anemia congénita $5,8 \%$, hidrocefalia y MC múltiples 3,9\%, MC tubo digestivo y renal 3,5\%, MC del Sistema nervioso y del corazón $2,6 \%$. 
Tabla 3. Prevalencia de malformaciones congénitas en madres adolescentes. Instituto Nacional Materno Perinatal - 2018

\begin{tabular}{|c|c|c|c|}
\hline & Malformaciones congénitas & $\mathbf{n}$ & $\%$ \\
\hline \multirow{6}{*}{ Adolescente } & Macrocefalia & 4 & $44.4 \%$ \\
\hline & Testículo no descendido, sin otra especificación & 2 & $22.2 \%$ \\
\hline & Hidrocéfalo congénito, no especificado & 1 & $11.1 \%$ \\
\hline & Otras anemias congénitas, no clasificadas en otra parte & 1 & $11.1 \%$ \\
\hline & Ausencia, atresia y estenosis congénita del recto, sin fístula & 1 & $11.1 \%$ \\
\hline & Total & 9 & $100.0 \%$ \\
\hline
\end{tabular}

En relación a madres adolescentes que tuvieron malformación congénita, se aprecia en la tabla 3 , que predominan la macrocefalia con $44,4 \%$, testículo no descendido $22,2 \%$, hidrocefalia $11,1 \%$

Tabla 4. Prevalencia de malformaciones congénitas en madres de edad media. Instituto Nacional Materno Perinatal - 2018

\begin{tabular}{|c|c|c|c|}
\hline & Malformaciones congénitas & $\mathbf{n}$ & $\%$ \\
\hline \multirow{36}{*}{ Edad media } & Otros síndromes de malformaciones congénitas debidos a causas exógenas conocidas & 14 & $15.4 \%$ \\
\hline & Malformación congénita del riñón, no especificada & 10 & $11.0 \%$ \\
\hline & Otras anemias congénitas, no clasificadas en otra parte & 7 & $7.7 \%$ \\
\hline & Macrocefalia & 7 & $7.7 \%$ \\
\hline & Hidrocéfalo congénito, no especificado & 4 & $4.4 \%$ \\
\hline & Malformación congénita del sistema nervioso, no especificada & 3 & $3.3 \%$ \\
\hline & Malformación congénita del corazón, no especificada & 3 & $3.3 \%$ \\
\hline & Síndrome de Down, no especificado & 3 & $3.3 \%$ \\
\hline & Testículo no descendido, sin otra especificación & 3 & $3.3 \%$ \\
\hline & Riñón poliquístico, autosómico recesivo & 3 & $3.3 \%$ \\
\hline & Otras malformaciones congénitas especificadas del (de los) miembro(s) & 3 & $3.3 \%$ \\
\hline & Otras deformidades congénitas de los pies & 2 & $2.2 \%$ \\
\hline & Malformaciones congénitas múltiples, no clasificadas en otra parte & 2 & $2.2 \%$ \\
\hline & Fisura del paladar duro y del paladar blando con labio leporino bilateral & 2 & $2.2 \%$ \\
\hline & Ausencia, atresia y estenosis congénita del duodeno & 2 & $2.2 \%$ \\
\hline & Ausencia, atresia y estenosis congénita del ano, sin fístula & 2 & $2.2 \%$ \\
\hline & Hipospadias, no especificada & 2 & $2.2 \%$ \\
\hline & Hidronefrosis congénita & 2 & $2.2 \%$ \\
\hline & Hidrocele congénito & 1 & $1.1 \%$ \\
\hline & Conducto arterioso permeable & 1 & $1.1 \%$ \\
\hline & Hemangioma, de cualquier sitio & 1 & $1.1 \%$ \\
\hline & Anemia congénita debida a pérdida de sangre fetal & 1 & $1.1 \%$ \\
\hline & Microcefalia & 1 & $1.1 \%$ \\
\hline & Atresia de los agujeros de Magendie y de Luschka & 1 & $1.1 \%$ \\
\hline & Malformaciones congénitas del cuerpo calloso & 1 & $1.1 \%$ \\
\hline & Otras anomalías hipoplásicas del encéfalo & 1 & $1.1 \%$ \\
\hline & Espina bífida lumbar sin hidrocéfalo & 1 & $1.1 \%$ \\
\hline & Espina bífida & 1 & $1.1 \%$ \\
\hline & Labio leporino & 1 & $1.1 \%$ \\
\hline & Ausencia, atresia y estenosis congénita del recto, sin fístula & 1 & $1.1 \%$ \\
\hline & Testículo no descendido, unilateral & 1 & $1.1 \%$ \\
\hline & Hiperplasia renal y riñón gigante & 1 & $1.1 \%$ \\
\hline & Otras deformidades congénitas de la cadera & 1 & $1.1 \%$ \\
\hline & Artrogriposis múltiple congénita & 1 & $1.1 \%$ \\
\hline & Malformación congénita de miembro(s), no especificada & 1 & $1.1 \%$ \\
\hline & Total & 91 & $100.0 \%$ \\
\hline
\end{tabular}


Como se aprecia en la tabla 4, la prevalencia de MC en edad reproductiva media, predominan otros síndromes de malformaciones congénitas de causas exógenas con $15,4 \%$, seguida $\mathrm{MC}$ renal $11 \%$; anemia congénita y macrocefalia 7,7\%; hidrocefalia 4,4\%; MC del sistema nervioso, cardiovascular, síndrome de Down, testículo no descendido, riñon, y de miembros en 3,3\%; entre otras.

Tabla 5. Prevalencia de malformaciones congénitas en edad materna avanzada. Instituto Nacional Materno Perinatal - 2018

\begin{tabular}{|c|c|c|c|}
\hline & Malformaciones congénitas & $\mathbf{n}$ & $\%$ \\
\hline \multirow{22}{*}{$\begin{array}{c}\text { Edad } \\
\text { materna } \\
\text { avanzada }\end{array}$} & Síndrome de Down, no especificado & 14 & $26.4 \%$ \\
\hline & $\begin{array}{l}\text { Otros síndromes de malformaciones congénitas debidos a causas exógenas } \\
\text { conocidas }\end{array}$ & 8 & $15.1 \%$ \\
\hline & Malformaciones congénitas múltiples, no clasificadas en otra parte & 4 & $7.5 \%$ \\
\hline & Fisura del paladar con labio leporino & 3 & $5.7 \%$ \\
\hline & Ausencia, atresia y estenosis congénita del recto, sin fístula & 3 & $5.7 \%$ \\
\hline & Otras malformaciones congénitas del hígado & 3 & $5.7 \%$ \\
\hline & Macrocefalia & 3 & $5.7 \%$ \\
\hline & Malformación congénita del riñón, no especificada & 2 & $3.8 \%$ \\
\hline & Hidrocele congénito & 1 & $1.9 \%$ \\
\hline & Hidrocéfalo congénito, no especificado & 1 & $1.9 \%$ \\
\hline & Malformación congénita del sistema nervioso, no especificada & 1 & $1.9 \%$ \\
\hline & Malformación congénita del corazón, no especificada & 1 & $1.9 \%$ \\
\hline & Otras anemias congénitas, no clasificadas en otra parte & 1 & $1.9 \%$ \\
\hline & Malformaciones congénitas del cuerpo calloso & 1 & $1.9 \%$ \\
\hline & Espina bífida & 1 & $1.9 \%$ \\
\hline & Fisura del paladar, sin otra especificación & 1 & $1.9 \%$ \\
\hline & Hidronefrosis congénita & 1 & $1.9 \%$ \\
\hline & Pie cavus & 1 & $1.9 \%$ \\
\hline & Deformidad congénita de la columna vertebral & 1 & $1.9 \%$ \\
\hline & Acondroplasia & 1 & $1.9 \%$ \\
\hline & Soplos cardíacos benignos o inocentes & 1 & $1.9 \%$ \\
\hline & Total & 53 & $53.0 \%$ \\
\hline
\end{tabular}

Según se aprecia en la tabla 5 en edad materna avanzada de 53 casos estudiados, predomina el Síndrome de Down con $26,4 \%$, seguida de otros síndromes de malformaciones congénitas debidas a causas exógenas con 15,1\%; malformaciones múltiples con $7,1 \%$; fisura del paladar con labio leporino ; MC recto, hígado y macrocefalia con $5,7 \%$ ; y MC del riñon con 3,8\%. 
Figura 1. Curva de supervivencia de Recien nacidos con malformaciones congénitas según sexo

\section{Curva de Supervivencia}

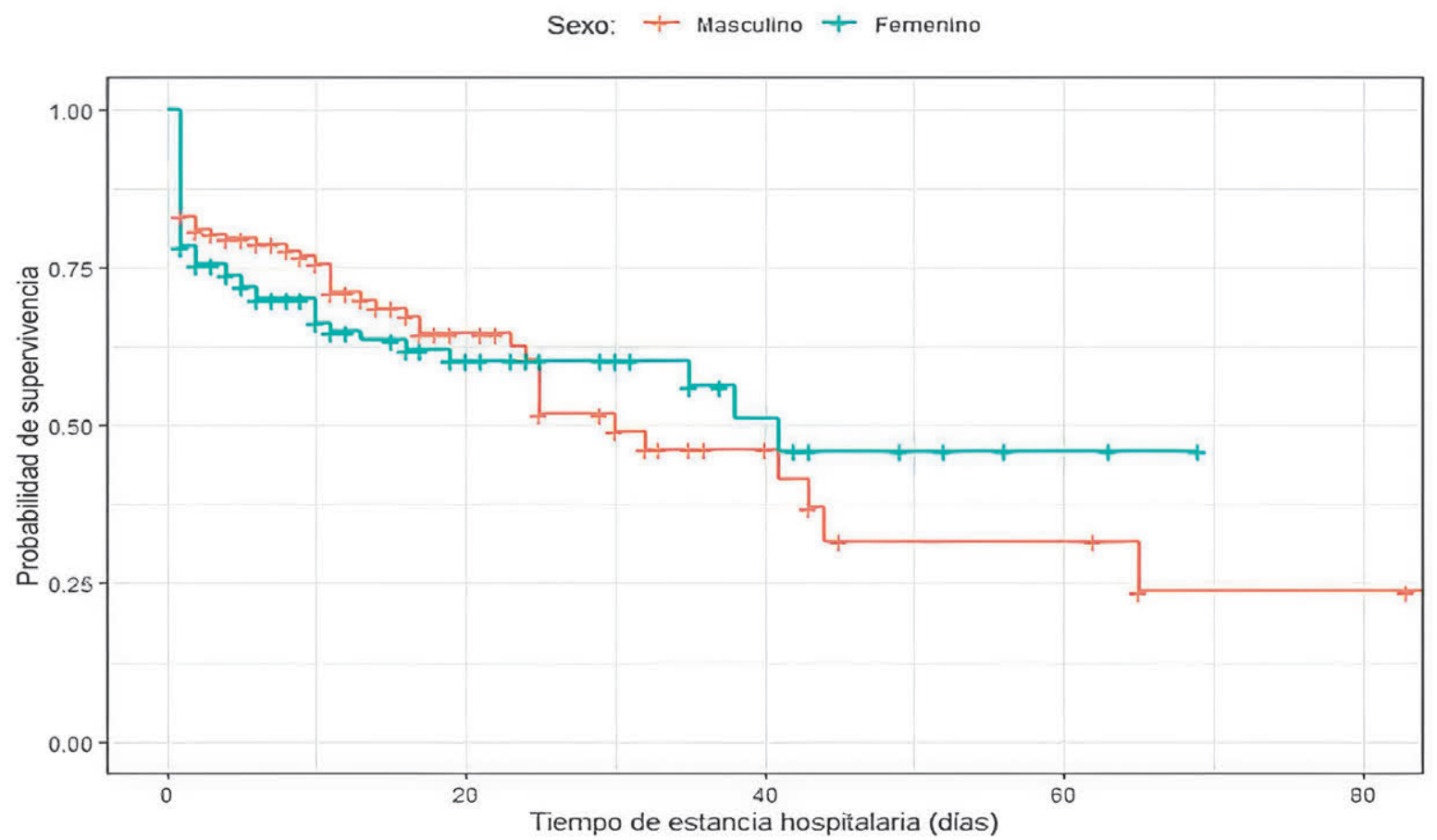

Según figura 1 la supervivencia de $\mathrm{RN}$ con $\mathrm{MC}$ según sexo a partir de 20 días de estancia hospitalaria el sexo femenino sobrevive mayor que el sexo masculino alcanzando por encima del $60 \%$ de probabilidad de supervivencia; y esto se mantiene cuando sobrepasa los 40 días donde la supervivencia alcanza por encima del $45 \%$.

Figura 2. Curva de supervivencia de recien nacidos con malformaciones congénitas según edad materna

\section{Curva de Supervivencia}

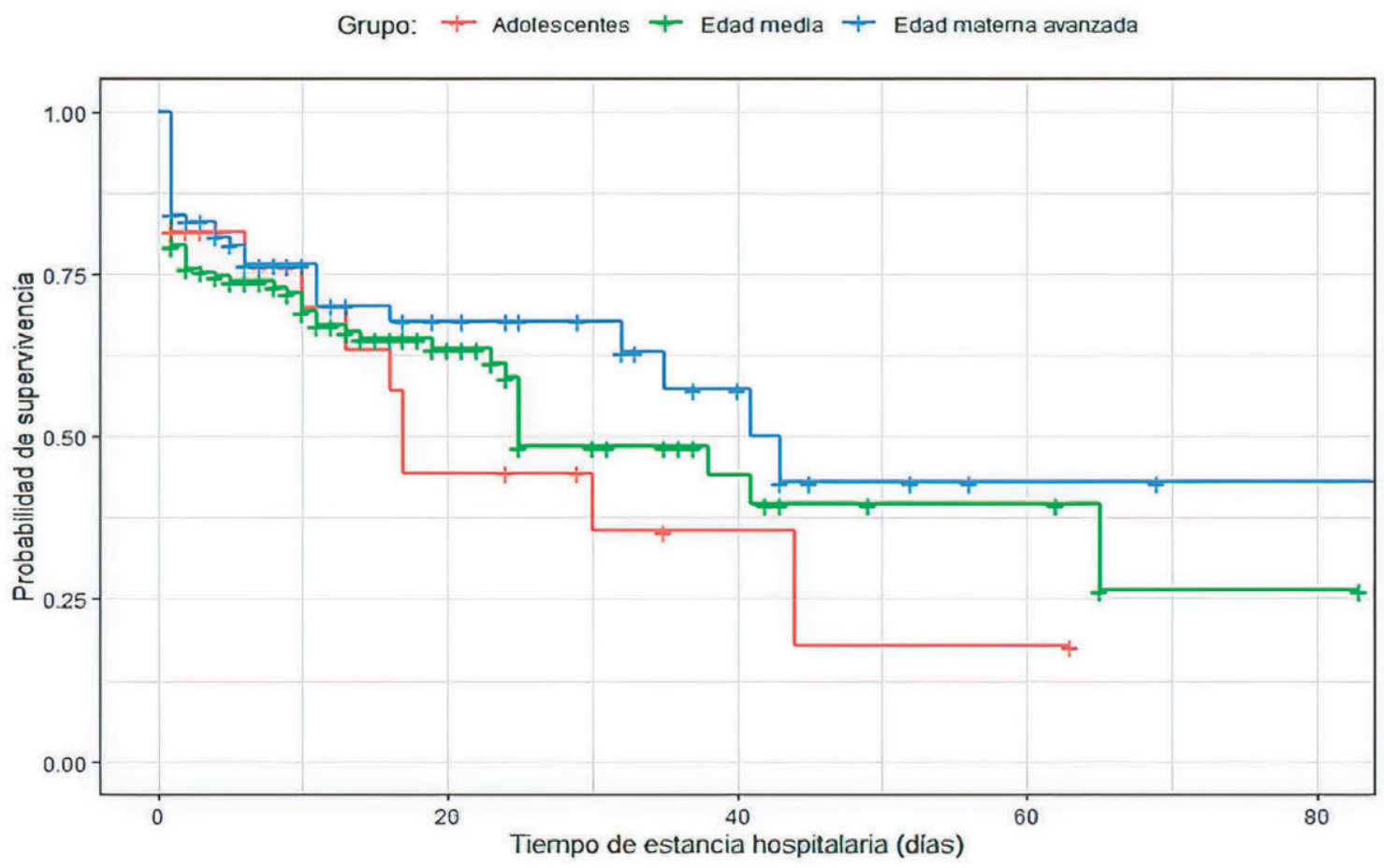


Según Figura 2 la supervivencia de RN con MC según grupo etario a partir de 15 días de estancia hospitalaria los $\mathrm{RN}$ de madres de edad media y edad materna avanzada sobreviven mayor que de las madres adolescentes alcanzando por encima del $62 \%$ de probabilidad de supervivencia; y esto se mantiene cuando sobrepasa los 40 días donde la supervivencia alcanza por aproximadamente sobre el $42 \%$.

\section{DISCUSIÓN}

En el Instituto Nacional Materno Perinatal durante el periodo de estudio se encontró prevalencia de 1,9\% MC que es similar al reporte de Fonseca $\mathrm{R}$ et $\mathrm{al}^{4}$ quienes mencionan entre $2-5 \%$ de nacimientos. Asimismo, la malformación congénita ocupa el primer lugar como causa de mortalidad en etapa neonatal con una tasa de mortalidad neonatal específica de MC de 8,1×1000 nacidos vivos; la que coincide con reportes de la OMS ${ }^{1}$.

En el presente estudio, la mayoría de casos de MC se encontró en edad media normal con $62,4 \%$ de casos seguida de edad materna avanzada en $29,7 \%$; la cual coincide con los estudios de Vázquez-Martínez $\mathrm{V}$ y col ${ }^{18}$ donde la media de la edad materna fue de 28,7 y al realizar el análisis según grupos de edades se observó que dentro del grupo de malformados resultó más frecuente la edad materna entre 20 y 35 años con el $67,6 \%$; y cuando ajustaron en función de la cantidad de recién nacidos vivos (RNV) según los grupos de edades, se observó que la edad materna donde más frecuentemente aparecen las malformaciones de los RNV es en edad materna avanzada con una tasa de 17,5/1000 RNV; y observaron que las madres con edad superior a 35 años tienen 2,37 veces más riesgo de tener un recién nacido vivo con malformación congénita que aquellas con edad inferior.

La identificación de factores de riesgo prenatales como antecedentes familiares o de un hijo previo con malformaciones congénitas, la falta de suplemento de ácido fólico antes y/o durante el embarazo, la exposición materna a plaguicidas entre otros, constituyen un componente fundamental para la consejería y por ende planificación del embarazo y/o nacimiento del niño; tal como señala Ojeda $\mathrm{L}$ y $\mathrm{col}^{8}$ las anomalías congénitas más frecuentemente detectadas fueron los defectos del tubo neural y las malformaciones cardiacas entre otras, dato que coincide a lo publicado en la literatura internacional ${ }^{4}$. En el presente estudio las malformaciones congénitas prevalentes según tipo de $\mathrm{MC}$, en madres adolescentes predominaron macrocefalia con $44,4 \%$, testículo no descendido $22,2 \%$, hidrocefalia $11,1 \%$, entre otras; en edad media, otros síndromes de malformaciones congénitas de causas exógenas con 15,4\%, seguida MC renal $11 \%$; anemia congénita y macrocefalia $7,7 \%$; entre otras; $y$ en edad materna avanzada predominaron el Síndrome de Down con $26,4 \%$, seguida de otros síndromes de malformaciones congénitas debidas a causas exógenas con $15,1 \%$; malformaciones múltiples con 7,1\%; entre otras. Nazer $\mathrm{HJ}$ et al ${ }^{19}$ tras análisis de 18 años por ECLAMC, encontró aumento de la prevalencia de algunas malformaciones como el síndrome de Down, la polidactilia, la anotia-microtia, la sindactilia y el paladar hendido y disminución la de prevalencia de espina bífida y anencefalia.

Por otro lado, de acuerdo con los criterios de presentación de la información del ICBDSR de México ${ }^{20-22}$, las principales causas de prevalencia de malformaciones congénitas, en orden descendente, fueron las siguientes: criptorquidia con 6,97 por 10,000 nacimientos; labio hendido con o sin paladar hendido, 5,22 por 10,000 nacimientos; polidactilia, 4,31 por 10,000 nacimientos; síndrome de Down, 3,55 por 10,000 nacimientos; gastrosquisis, 1,91 por 10,000 nacimientos.

En relación al factor de riesgo edad materna, podemos señalar que las MC se pueden presentar en cualquier etapa de la vida de la mujer, tal como se encontró en el estudio, prevalecieron en edad media y edad materna avanzada; que coincide con reporte de Ojeda $\mathrm{L}$ y $\mathrm{col}^{8}$ quienes mencionan que la edad materna con una media de 27 años, no se asoció como factor de riesgo de $\mathrm{MC}$; aunque, en otros estudios como Zarate $\mathrm{A}$ y $\mathrm{col}^{23}$ y Zavaleta $\mathrm{M}$ y col ${ }^{24}$ la edad materna adolescente o edad materna avanzada fueron factores de riesgo asociados estadísticamente a malformaciones congénitas.

Varios estudios como de Roberts JR et al ${ }^{9} y$ de Rojas A y $\mathrm{col}^{10}$ a lo largo de la historia han intentado asociar la presencia de malformaciones congénitas con factores de riesgo, algunos mencionan como factores de riesgo evidenciados: edad materna avanzada, bajo peso y talla para la edad gestacional. Otros autores como Canals CA et a ${ }^{25}$ mencionan además que la restricción del crecimiento fetal, antecedentes de malformaciones congénitas en la familia, los factores físicos y enfermedades agudas de la madre en el primer trimestre del embarazo, fueron factores de riesgo significativos para las malformaciones congénitas.

Es importante precisar que en el presente estudio se encontró mayor prevalencia del Síndrome de Down en mujeres con edad materna avanzada; tal como señala Vázquez-Martínez $\mathrm{V}$ y $\mathrm{col}^{18}$ que al analizar la edad materna, coinciden los resultados con los de otros autores ${ }^{26}$, quienes plantean que el riesgo de tener un hijo malformado aumenta con la edad, sobre todo en relación con el síndrome de Down y otras trisomías como la 18 y la 13. Una de las explicaciones que se han dado para relacionar el aumento de la frecuencia de este tipo de aberraciones cromosómicas con la edad materna avanzada, es el aumento de la no disyunción en los ovocitos de mujeres en edad materna avanzada, que permanecen en estado de dictioteno, desde la etapa fetal hasta esta época ${ }^{27,28}$

El resultado perinatal en el presente estudio demostró que el parto pretérmino conjuntamente con bajo peso al nacer en niños con malformación congénita tuvieron significancia estadística; tal como señalan Nazer HJ et 
al ${ }^{19}$ la prematurez y el bajo peso al nacer tuvieron mayor frecuencia en los casos, al igual que en otros estudios similares; sin embargo con un valor de $p$ no significativo.

Es preciso señalar que durante el presente estudio se encontró limitaciones en la identificación principal con la codificación internacional (CIE 10) de malformación congénita que se registran en la oficina de estadística institucional que dificultó sistematizar en prevalencia por órganos o sistemas afectados. Por otro lado, por ser un estudio retrospectivo algunos datos como antecedentes de malformaciones previas no se encontraron registros así como otros factores como consumo de medicamentos o drogas durante el embarazo.

Finalmente, señalar que en el presente estudio de malformaciones congénitas, la probabilidad de supervivencia de recién nacida de sexo femenino fue mayor que el masculino alcanzando por encima del $60 \%$ después de 20 días; y los recién nacidos de madres de edad media normal y edad materna avanzada sobreviven mayor que de las madres adolescentes alcanzando por encima del $62 \%$ después de 15 días de nacido.

En conclusión, nuestros hallazgos indican que el recién nacido pretérmino, bajo peso al nacer y puntuación de Apgar bajo al nacer tuvieron asociación estadística significativa; asimismo, el Síndrome de Down fue la MC predominante en edad materna avanzada.

Financiamiento: Fondo Concursable de Investigación 2019 de la Universidad

Norbert Wiener

Conflicto de interés: Los autores declaran no tener algún conflicto de intereses.

\section{REFERENCIAS BIBLIOGRÁFICAS}

1. World Health Organization. Anomalías congénitas. Nota descriptiva. 7 de septiembre de 2016. Disponible en https:// www.who.int/es/news-room/fact-sheets/detail/congenitalanomalies.

2. Organización Panamericana de Salud (OPS). Organización Mundial de Salud (OMS). Boletín informativo. Malformaciones congénitas. Junio 2015. Disponible

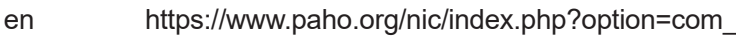
docman\&view=download\&category_slug=datos $-y$ estadisticas\&alias=711-boletin-informativo-malformacionescongenitas\& $\mathbf{I t e m i d}=235$

3. Instituto Nacional Materno Perinatal. Boletín epidemiológico 2018. Disponible en https://www.inmp.gob.pe/institucional/ boletinepidemiologico/1421335605? pagina $=1$

Fonseca R, Mir R, Irala S, Navarro E, Ortigosa M, Céspedes E, Caballero Bareiro C. Conocimientos de la Etiología y los Factores de Riesgo de los Defectos Congénitos. Pediatría. Pediatr. (Asunción) 2008; 35(2):95-100.

Instituto Nacional de Salud. Vigilancia y análisis del riesgo en salud pública. Protocolo de vigilancia en salud pública. Defectos congénitos. Colombia, 2018.

Christianson A, Howson CP, Modell B. Global Report on Birth Defects: The Hidden Toll of Dying and Disabled Children.
White Plains, New York. 2006

4. Rojas M, Walker L. Malformaciones Congénitas: Aspectos Generales y Genéticos. Int J Morphol. 2012;30(4):1256-1265. Disponible en https://scielo.conicyt.cl/pdf/ijmorphol/v30n4/ art03.pdf.

Ojeda L, Leite S. Factores de riesgo prenatales y su asociación a

5. malformaciones congénitas en un Hospital Universitario de Referencia. Pediatr. (Asunción). 2018; 45(1):08-16

Roberts JR, Karr CJ. Pesticide exposure in children. Pediatrics. 2012; 130(6):e1765-88. doi: 10.1542/peds.20122758.

Rojas A, Ojeda ME, Barraza X. Malformaciones congénitas y exposición a pesticidas. Rev. Méd. Chile. 2000;128:399-404.

6. Loane M, Morris JK, Addor MC, Arriola L, Budd J, Doray B, Garne E, Gatt M, Haeusler M, Khoshnood B, Klungsøyr Melve K, Latos-Bielenska A, McDonnell B, Mullaney C, O'Mahony M, Queisser-Wahrendorf A, Rankin J, Rissmann A, Rounding C, Salvador J, Tucker D, Wellesley D, Yevtushok $\mathrm{L}$, Dolk H. Twenty-year trends in the prevalence of Down syndrome and other trisomies in Europe: impact of maternal age and prenatal screening. Eur J Hum Genet. 2013;21(1):2733. doi: 10.1038/ejhg.2012.94.

Jacobs M, Cooper S-A, McGowan R, Nelson SM, Pell JP. Pregnancy outcome following prenatal diagnosis of chromosomal anomaly:a record linkage study of 26,261 pregnancies. PLoS ONE. 2016;11(12):e0166909.

7. de Graaf G, Buckley F, Dever J, Skotko BG. Estimation of live birth and population prevalence of Down syndrome in nine U.S. states. Am J Med Genet A. 2017;173(10):2710-2719. doi: 10.1002/ajmg.a.38402.

8. Mitchell LE, Adzick NS, Melchionne J, Pasquariello PS, Sutton LN, Whitehead AS. Spina bifida. Lancet. 2004;364(9448):1885-95.

9. Khoshnood B, Loane M, de Walle H, Arriola L, Addor MC, Barisic I, Beres J, Bianchi F, Dias C, Draper E, Garne E, Gatt M, Haeusler M, Klungsoyr K, Latos-Bielenska A, Lynch C, McDonnell B, Nelen V, Neville AJ, O'Mahony MT, QueisserLuft A, Rankin J, Rissmann A, Ritvanen A, Rounding C, Sipek A, Tucker D, Verellen-Dumoulin C, Wellesley D, Dolk H. Long term trends in prevalence of neural tube defects in Europe: population based study. BMJ. 2015;351:h5949. doi: 10.1136/ bmj.h5949.

10. Grillo E, da Silva RJ. Neural tube defects and congenital hydrocephalus. Why is prevalence important?. J Pediatr (Rio J). 2003;79(2):105-6.

11. Instituto Nacional Materno Perinatal. Boletín estadístico 2018. Disponible en https://www.inmp.gob.pe/institucional/ boletines-estadisticos/1422371837

12. Vázquez-Martínez $V$, Torres-González $C$, Dueñas $A$, Vázquez G, Díaz D, de-la-Rosa-López R. Malformaciones congénitas en recién nacidos vivos. Medisur [revista en Internet]. 2013 [citado 2019 Dic 13]; 12(1):[aprox. 8 p.].Disponible en: http:// medisur.sld.cu/index.php/medisur/article/view/2639

13. Nazer $H$ J, Cifuentes $O$ L. Prevalence of congenital malformations at birth in Chilean maternity hospitals. Rev Med Chil. 2014;142(9):1150-6. doi: 10.4067/S003498872014000900009

14. Navarrete-Hernández E, Canún-Serrano $S$, ValdésHernández J, Reyes-Pablo AE. Malformaciones congénitas 
al nacimiento: México, 2008-2013. Bol Med Hosp Infant Mex. 2017;74(4):301-308

15. International Clearinghouse for Birth Defects Surveillance and Research. Synopsis of Contributing Monitoring Systems. Austin: Annual Report. 2013.

16. Bases de datos de nacimientos y muertes fetales. Dirección de General de Información en Salud. México: Secretaria de Salud; 2016.

Zarate A, Gracia G, Zarante I. Evaluación de factores de riesgo asociados con malformaciones congénitas en el programa de vigilancia epidemiológica de malformaciones congénitas (ECLAMC) en Bogotá entre 2001 y 2010. Universitas Médica. 2012; 53(1): 11-25..

Zavaleta M, Cortegana-Aranda J, Zavaleta-Gutierrez F, Ocampo-Rugel C, Estrada-Alva L. Factores maternos asociados a malformaciones congénitas en recién nacidos de un Hospital de Trujillo, Perú. Rev. Cuerpo Méd. 2016; 9(2).

Canals CA, Cavada C G, Nazer H J. Identification of risk factors for congenital malformations. Rev Med Chil. 2014;142(11):1431-9. doi: 10.4067/S003498872014001100010 .

Vázquez-Martínez V, Torres González C, González Jiménez G, Del Sol Y, López Rodríguez del Rey AM, Barberis Pérez G. Malformaciones congénitas mayores. Factores de riesgo relevantes. Cienfuegos 2000-2005. Medisur [revista en Internet]. 2008 [ cited 4 Mar 2013 ] ;6 ( 1 ) : [ aprox . 8 p ] .
Available from: http://www.medisur.sld.cu/index.php/medisur/ article/view/346.

17. Ministerio de Salud Pública. Anuario Estadístico de Salud 2011 [Internet]. La Habana: MINSAP; 2012. [ cited 4 Mar 2013 ] Available from: http://www.one.cu/aec2011/esp/20080618_ tabla_cuadro.htm.

18. Marcheco B. El Programa Nacional de Diagnóstico, Manejo y Prevención de Enfermedades Genéticas y Defectos Congénitos de Cuba: 1981-2009. Rev Cubana Genet Comunit [revista en Internet]. 2009 [ cited 18 Ene 2013 ] ;3 (2-3): [aprox. 13p]. Available from:

19. http://bvs.sld.cu/revistas/rcgc/v3n2_3/rcgc1623010 esp.htm.

\section{Correspondencia:}

Félix Dasio Ayala Peralta.

Dirección: Jr. Maracaibo N²153 San Martín de Porres.Lima-Perú.

Correo electrónico: fayala1401@hotmail.com;fayala@wiener.edu.pe 